\title{
连续纤维增强陶瓷基复合材料微观力学研究进展
}

\author{
刘海蹈 ${ }^{1}$, 杨玲伟 ${ }^{2}$, 韩 爽 ${ }^{1}$ \\ (1. 国防科技大学 新型陶瓷纤维及其复合材料重点实验室, 长沙 410073; 2. 西班牙 IMDEA 材料研究所, 马德里 \\ 28906) \\ 摘 要: 微观力学参数是构建连续纤维增强陶瓷基复合材料(CFRCMCs)组分、微观结构和宏观力学性能的桥梁, 但 \\ 受限于 CFRCMCs 的脆性和微观力学参数测试水平, 微观力学研究工作进展缓慢。随着基于纳米压痕的微观力学测 \\ 试技术和基于聚焦离子束微观测试样品制备技术的飞速发展, 近年来 CFRCMCs 的微观力学研究工作取得显著进 \\ 步。本文结合国防科技大学刘海蹈课题组的研究工作, 重点对 CFRCMCs 组分的原位模量、断裂韧性以及界面结合 \\ 强度的测试方法和典型应用进行了讨论, 最后举例说明了基于微观力学参数的 CFRCMCs 宏观力学行为的预判方法。
} 关 键 词: 陶瓷基复合材料; 微观力学; 纳米压痕; 聚焦离子束; 综述

中图分类号: TB323 文献标识码: A

\section{Research Progress on Micro-mechanical Property of Continuous Fiber-reinforced Ceramic Matrix Composites}

\author{
LIU Hai-Tao ${ }^{1}$, YANG Ling-Wei ${ }^{2}$, HAN Shuang ${ }^{1}$
}

(1. Science and Technology on Advanced Ceramic Fibers and Composites Laboratory, National University of Defense Technology, Changsha 410073, China; 2. IMDEA Materials Institute, Madrid 28906, Spain)

\begin{abstract}
Localized mechanical properties of composite components (fiber, matrix, and interface) are critical parameters bridging the composition, microstructure and macro-mechanical performance of continuous fiber-reinforced ceramic matrix composites (CFRCMCs). However, they are difficult to be acquired and decoupled from bulk composites based on the traditional macro-mechanical testing techniques, due to their limited testing volumes and complex heterogeneous composite structures. The above questions has been solved recently by novel nano/micro mechanical testing and focused ion beam milling (FIB) techniques, which provide powerful tools to quantify the micro-mechanical properties of CFRCMCs. In this paper, recent progress in micro-mechanical properties of CFRCMCs was firstly reviewed, with special emphasis on the in-situ modulus and toughness of ceramic fibers and matrix, and the shear property of fiber/matrix interface. Following that, a criterion based on the He-Hutchinson cracking model was proposed to predict the macro mechanical performance of CFRCMCs by using those micro-mechanical parameters.
\end{abstract}

Key words: ceramic matrix composites; micro-mechanics; nanoindentation; focused ion beam; review

连续纤维增强陶瓷基复合材料(CFRCMCs)具 有耐高温、高比强度、高比模量、耐腐蚀等优异特 性, 特别是高效增韧相连续陶瓷纤维的引入，克服 了本体陶瓷材料的固有脆性，使其在航空航天发动
机热端部件、地面燃气轮机、高速飞行器热结构部 件和热防护系统、高速刹车等领域具有广阔的应用 前景, 并已付诸应用 ${ }^{[1-5]}$ 。

经过几十年的发展, CFRCMCs 在工艺与性能

收稿日期: 2017-08-30; 收到修改稿日期：2017-10-26

基金项目: 国家自然科学基金(51202291) National Natural Science Foundation of China (51202291)

作者简介: 刘海蹈(1981-), 男, 副研究员. E-mail: xzddlht@163.com 
研究等方面取得了丰硕成果, 但到目前为止, 仍然 难以实现精确的力学性能仿真和力学行为预判, CFRCMCs 组分、微观结构和宏观力学性能间本构 关系尚未精确构建, 这主要是因为 CFRCMCs 中各 组分原位微观力学参量的缺失。CFRCMCs 中各组 分的原位微观力学参量(主要包括纤维与基体的模 量、㓞性, 界面结合强度等)既决定了 CFRCMCs 的 宏观力学性能, 又是宏观力学数值仿真的关键输入 参量 ${ }^{[6-8]}$ 。长久以来, CFRCMCs 中各组分微观力学 参量的测量一直是难点问题, 这一方面由于陶瓷材 料固有的脆性使小尺度微观力学测试样品制备困难, 另一方面由于微观力学参数测试手段与理论的不完 善 ${ }^{[9]}$, 导致 CFRCMCs 微观力学研究工作进展相对 缓慢。

近年来, 随着以纳米压痕为代表的纳米力学测 量技术和以聚焦离子束(FIB)为代表的微纳加工技 术的快速发展, CFRCMCs 原位微观力学研究工作 取得显著进步, 并在 CFRCMCs 宏观力学性能研究 工作中发挥了重要作用 ${ }^{[10-23]}$ 。本文将结合国防科技 大学刘海蹈课题组的研究工作评述 CFRCMCs 中微 观力学的最近研究进展情况, 重点对 CFRCMCs 中 纤维与基体的原位模量、断裂韧性, 界面结合强度 的测试方法进行分析, 并对基于微观力学参量的 CFRCMCs 宏观力学行为的预判应用进行讨论。

\section{1 纤维与基体原位模量}

纤维与基体是 CFRCMCs 的重要组成部分, 其 原位模量、断裂韧性等微观力学参量决定了复合材 料的宏观力学性能。同时, 纤维与基体的微观力学 参量又可以直观反映出工艺对 CFRCMCs 组分性能 的影响, 比如基体的烧结特性、纤维的损伤情况等。 此外, 纤维与基体的微观力学参量作为 CFRCMCs 的 基础物理量, 也是进行 CFRCMCs 力学仿真的基础。

\section{1 纤维与基体原位模量测量}

\subsection{1 测量原理}

材料微区模量的测量是纳米压痕测试系统的基 本功能。采用纳米压痕测试技术可以测量 CFRCMCs 中纤维与基体的原位模量 ${ }^{[24-27]}$, 测量过程简单易操 作, 可以真实地反映 CFRCMCs 中纤维与基体的原 位力学参量信息, 相对于传统通过纤维与基体宏观 力学测试方法获取的力学参量更加准确。测量过程 如下: 对 CFRCMCs 进行抛光处理, 在纳米压痕成 像系统辅助下, 分别定位纤维与基体区域, 采用 Berkovich 压头进行加载, 根据 Oliver-Pharr 定律计算
测量样品的弹性模量 ${ }^{[24]}$.

$$
E=\left(1-v^{2}\right)\left(\frac{1}{E_{\mathrm{r}}}-\frac{1-v_{\mathrm{i}}^{2}}{E_{\mathrm{i}}}\right)^{-1}
$$

$E_{\mathrm{r}}$ 为约化模量, $v$ 为泊松比, $E_{\mathrm{i}}$ 和 $v_{\mathrm{i}}$ 分别为压头的弹 性模量和泊松比。

\subsection{2 纤维原位模量测量}

与单一组分材料模量测试不同, 由于 CFRCMCs 中小尺度纤维容易受到周边界面与基体力学环境的 影响, 采用纳米压痕技术测试纤维原位模量时需要 特别关注测量值与压头压入深度的关系, 对于界面 结合强度较低的样品, 压头加载过程如果造成界面 脱粘情况, 将导致测量的模量数据随压入深度的增 加而发生变化。

以国防科技大学刘海蹈课题组前期研究的溶胶凝胶工艺制备的连续铝硅酸盐 $(\mathrm{AS})$ 纤维增强氧化硅 $\left(\mathrm{AS}_{\mathrm{f}} / \mathrm{SiO}_{2}\right)$ 复合材料为例 ${ }^{[10]}$, 采用纳米压痕测量的 不同烧结温度制备的 $\mathrm{AS}_{\mathrm{f}} / \mathrm{SiO}_{2}$ 复合材料中, $\mathrm{AS}$ 纤维 的模量与压入深度的关系如图 1(a)所示。可以看出, 除界面结合强度较高( $250 \mathrm{MPa})$ 的 $1200^{\circ} \mathrm{C}$ 制备的复 合材料外, 其它温度制备的界面结合强度较低的 $(\sim$ 几
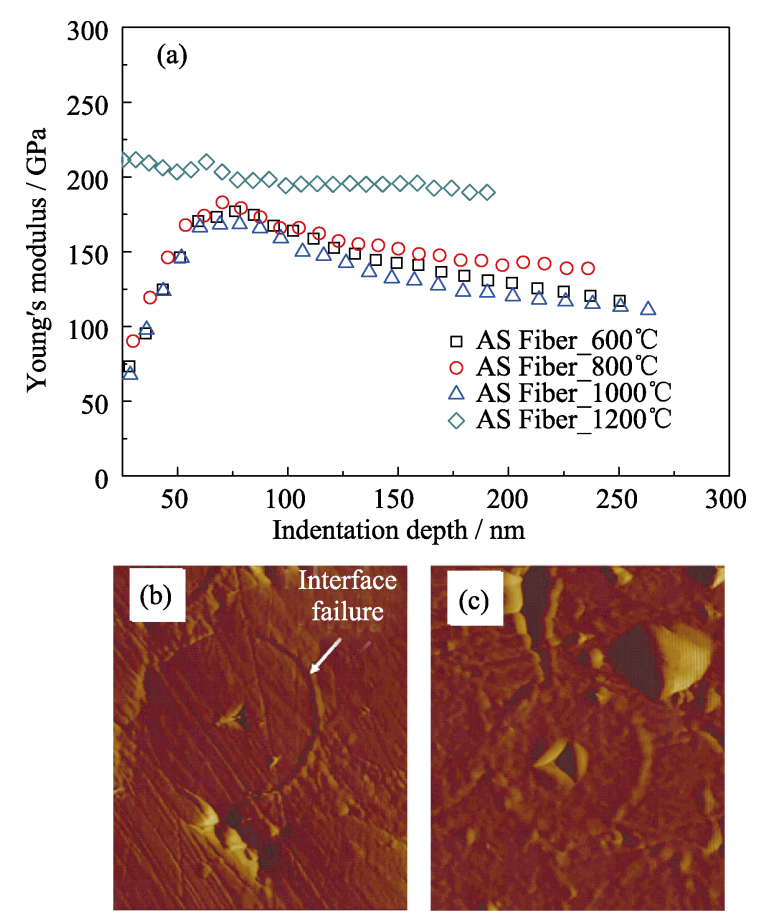

图 1 (a)不同温度制备的 $\mathrm{AS}_{\mathrm{f}} / \mathrm{SiO}_{2}$ 复合材料 $\mathrm{AS}$ 纤维模量与 压头压入深度关系; $600^{\circ} \mathrm{C}(\mathrm{b})$ 和 $1200^{\circ} \mathrm{C}(\mathrm{c})$ 制备的 $\mathrm{AS}_{\mathrm{f}} / \mathrm{SiO}_{2}$ 复合材料 AS 纤维纳米压痕测试后的 SPM(Scanning Probe Microscopy)照片 ${ }^{[10]}$

Fig. 1 (a) Young's modulus of the AS fiber in $\mathrm{AS}_{\mathrm{f}} / \mathrm{SiO}_{2}$ composites prepared at different temperatures as a function of penetration depth; SPM images of the nanoindentation imprints of $\mathrm{AS}_{\mathrm{f}} / \mathrm{SiO}_{2}$ composites fabricated at $600^{\circ} \mathrm{C}$ (b) and $1200^{\circ} \mathrm{C}$ (c) ${ }^{[10]}$ 
十兆帕)复合材料中 AS 纤维的模量均随着压入深度 的增加呈抛物线性变化，当压头压入深度约 $75 \mathrm{~nm}$ 时，模量达到最大值。这是由于当压入深度较小时， 测试的模量数据受纤维表面条件影响较大, 随着压 入深度的增加, 表面影响逐渐被消除; 当测试模量 达到最大值后，继续加载出现了测试模量下降的现 象，这主要是由较弱的界面结合造成的界面脱粘所 致(见图 1(b)), 此时压入深度不仅与压头压入纤维 的深度有关, 还受纤维位移影响。对于界面出现脱 粘的情况, Oliver-Pharr 定律失效, 测试模量的最大 值最接近于纤维模量的真实值。 $1200^{\circ} \mathrm{C}$ 制备的复合 材料由于具有较高的界面结合强度，在压头加载过 程中不会出现界面脱粘问题(见图 1(c)), 当压入深 度足以消除测试样品表面影响后, 测试模量值基本 不受压入深度影响, 可以代表测试样品的模量数据。

\subsection{3 基体原位模量测量}

由于在 CFRCMCs 截面很容易获取面积较大的 基体测试区域，因此相对于纤维，基体模量测量受 其它组分影响较小，使基体原位模量测量相对简 单。同样以不同温度制备的 $\mathrm{AS}_{\mathrm{f}} / \mathrm{SiO}_{2}$ 复合材料的 $\mathrm{SiO}_{2}$ 基体为例(见图 2) ${ }^{[10]}$, 可以发现, 当压头压入深 度达到一定值后，即消除测试样品表面影响后，基 体的测试模量值基本与压入深度无关。

\section{2 纤维与基体原位模量的典型应用}

纤维与基体的原位模量是 CFRCMCs 宏观力学 性能仿真与预判的必要参量, 同时也是纤维与基体 原位韧性、界面结合强度等微观力学参量测试过程 的必要输入参数, 这将结合后续内容展开讨论。

这里重点讨论纤维与基体原位模量数据所反映

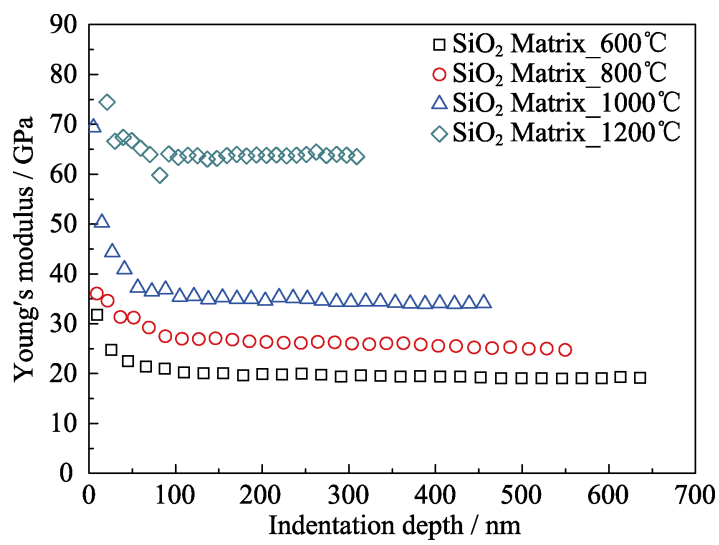

图 2 不同温度制备的 $\mathrm{AS}_{\mathrm{f}} / \mathrm{SiO}_{2}$ 复合材料 $\mathrm{SiO}_{2}$ 基体模量与 压头压入深度关系 ${ }^{[10]}$

Fig. 2 Young's modulus of the $\mathrm{SiO}_{2}$ matrix in $\mathrm{AS}_{\mathrm{f}} / \mathrm{SiO}_{2}$ composites prepared at different temperatures as a function of penetration depth ${ }^{[10]}$
的 CFRCMCs 组分与微观结构演变信息。同样以 $\mathrm{AS}_{\mathrm{f}} / \mathrm{SiO}_{2}$ 复合材料为例 ${ }^{[10]}$, 如图 1 所示, 当复合材 料的制备温度在 $600 \sim 1000^{\circ} \mathrm{C}$ 时，纤维的原位模量没 有明显差异, 均为 $170 \mathrm{GPa}$ 左右, 纤维在此温度范 围内未发生明显变化; 当制备温度达到 $1200^{\circ} \mathrm{C}$ 时, 纤维的模量增加至 $200 \mathrm{GPa}$, 在此温度下纤维发生 了晶化。以上结论与纤维物相以及单丝拉伸实验结 果相一致。类似的，如图 2 所示，随着制备温度的升 高, $\mathrm{SiO}_{2}$ 基体的模量显著增加，由 $600^{\circ} \mathrm{C}$ 制备温度时 的 $20 \mathrm{GPa}$ 上升至 $1200^{\circ} \mathrm{C}$ 时的 $60 \mathrm{GPa}$, 说明 $\mathrm{SiO}_{2}$ 基体的烧结程度显著增加。本课题组研究的不同先 驱体转化工艺(PIP)温度制备的 $\mathrm{SiC}_{\mathrm{f}} / \mathrm{SiC}$ 复合材料的 纤维与基体的原位模量也得到类似结论 ${ }^{[28]}$ 。因此, CFRCMCs 纤维与基体的原位模量可以准确地反映 出其随工艺参数的演变信息, 对于构建 CFRCMCs 工艺-结构-性能间关系, 指导 CFRCMCs 宏观力学 性能优化具有重要意义。

\section{2 纤维与基体原位断裂韧性}

与模量类似, 断裂韧性也是 CFRCMCs 中纤维 与基体的基础微观力学参数, 但相对模量, 原位断 裂韧性测量相对困难。长久以来, 存在采用纤维和 基体宏观力学方法测试断裂韧性, 并以其作为复合 材料各组分的原位断裂韧性数据的研究现象, 如纤

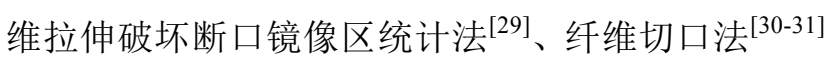
和基体单体陶瓷单边切口梁法 ${ }^{[12]}$ 等。这种宏观力学 性能测试方法无法准确体现制备工艺对 CFRCMCs 各 组分的性能影响, 无法做到与 CFRCMCs 各组分微 观结构严格一致，更无法体现 CFRCMCs 各组分的 力学环境(如残余应力等), 易造成数值仿真与理论 预判的错误结论。

\section{1 纤维与基体原位断裂韧性测量}

近年来，日趋完善的纳米力学测试方法和 FIB 微尺度样品制备方法促进了脆性材料原位断裂韧性 测试技术的快速发展，测试方法包括以下三种。

\subsection{1 尖压头压痕法(Indentation-based methods with pyramidal indenters)}

尖压头压痕法最先用于脆性材料原位断裂韧性 的测试，即采用尖压头对抛光的测试区域施加载荷, 使测试样品产生裂纹(见图 3), 然后通过测量在特 定载荷条件下的裂纹长度, 通过 LEM(Lawn-EvansMarshall)模型计算断裂韧性 $K_{\mathrm{IC}}{ }^{[18,32]}$. 


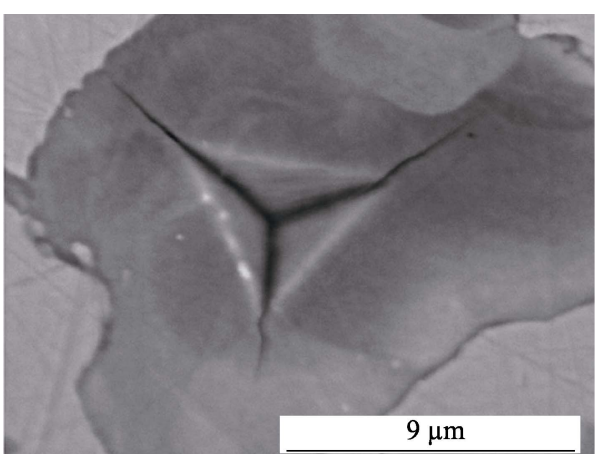

图 3 碳化物陶瓷涂层压痕裂纹 SEM 照片 ${ }^{[32]}$

Fig. 3 Radial cracks originating at the edges of a Berkovick indentation for carbide coating ${ }^{[32]}$

$$
K_{\mathrm{IC}}=\alpha \cdot \sqrt{\frac{E}{H}} \cdot \frac{P_{\max }}{c^{3 / 2}}
$$

式中 $\alpha$ 为 LEM 系数,一般取 $0.016 ; E$ 为弹性模量; $H$ 为硬度; $P_{\max }$ 为最大载荷; $c$ 为裂纹长度。其中 $E$ 和 $H$ 均采用纳米压痕方法测试。

由于 CFRCMCs 中容易获取面积相对较大的基 体测试区域，采用尖压头压痕法测量基体断裂韧性 时可使裂纹仅在基体区域扩展，避免纤维或界面对 裂纹传播路径的影响, 从而确保测试数据的有效 性。但此法测量纤维断裂韧性时存在较大问题, 这 是由于纤维尺度小, 难以将裂纹限制在纤维内部, 如果裂纹在纤维与基体界面发生贯穿或者偏转, 则 造成测试结果的失效。此外, 与纤维原位模量测试 过程存在类似的问题, 界面结合强度也会对纤维断 裂韧性的测量造成较大影响。再者, 由于尖压头压 痕法计算断裂韧性时需要统计裂纹长度, 这一过程 存在较大主观性，导致该方法测试精度不高。

\subsection{2 切口微梁法(Micro-cantilever beam method)}

为了克服尖压头压痕法测试多相材料断裂韧性 过程中易受其它组分影响的不足，人们发展了切口 微梁测试方法 ${ }^{[11,18,33]}$ 。单边切口微梁法首先用 FIB
制备出如图 4(a)所示的测试样品 ${ }^{[18]}$, 样品尺度为微 米量级。测试样品上有一预制微裂纹, 采用纳米压 痕对其加载, 直至样品断裂, 根据式(3)即可得到材 料的原位断裂韧性 ${ }^{[18]}$ :

$$
K_{\mathrm{IC}}=\sigma_{\mathrm{c}} \cdot \sqrt{\pi a} \cdot F\left(\frac{a}{b}\right)
$$

式中, $\sigma_{\mathrm{c}}$ 为断裂应力; $a$ 为裂纹长度; $F(a / b)$ 是与样品 尺寸相关的形状因子。Frazer 等 ${ }^{[11]}$ 采用此方法成功 测量了 $\mathrm{SiC}_{\mathrm{f}} / \mathrm{SiC}$ 复合材料中碳化硅基体的原位断裂 㓞性(见图 4(b) (c))。

通过以上分析可以发现，切口微梁法有效避免 了尖压头压痕法存在的裂纹长度统计主观性强、难 以测量小尺度纤维断裂韧性的不足, 但切口微梁法 测试样品较为复杂, 加工过程容易造成样品损伤, 特别是在切口位置, 会给裂纹传输过程带来较大影 响，因此切口微梁法操作复杂，不易实现。

\subsection{3 微柱法(Micro-pillar method)}

为了克服尖压头压痕法和切口微梁法的不足， 2014 年 Sebastiani 等 ${ }^{[17]}$ 提出了一种尖压头压微柱的 方法测试脆性材料的原位断裂韧性，这种方法最先 用于测试陶瓷涂层的断裂韧性，用以避免涂层基底 和残余应力的影响。具体测试过程为: 首先采用 FIB 制备直径为微米级、长径比 1 的微柱, 然后采用尖 压头对微柱加载使之开裂(见图 5), 根据载荷-位移 曲线, 采用式(4)即可得到材料的原位断裂韧性 ${ }^{[17]}$ :

$$
K_{\mathrm{IC}}=\gamma \frac{P_{\mathrm{c}}}{R^{3 / 2}}
$$

式中, $R$ 为微柱半径; $P_{\mathrm{c}}$ 为失效载荷; $\gamma$ 为与材料的弹 一塑性能相关的因子, 可以通过有限元方法计算得 到 ${ }^{[10,17-18,34]}$ 。

微柱法具有以下优点: (1)不必统计裂纹长度, 避免了主观性; (2)相对切口微梁法, 微柱不用预制 裂纹，制样相对简单，对样品损伤小; 3微柱法可
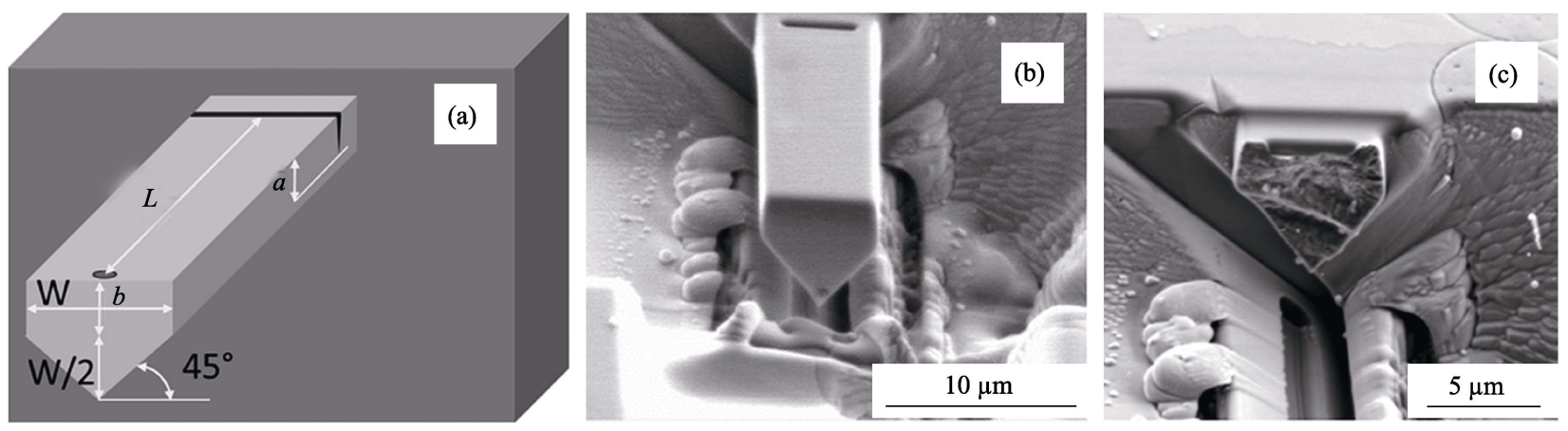

图 4 (a)单边切口微梁尺寸示意图; $\mathrm{SiC}_{\mathrm{f}} / \mathrm{SiC}$ 复合材料碳化硅基体单边切口微梁加载前(b)后(c)的 $\mathrm{SEM}$ 照片 ${ }^{[11,18]}$

Fig. 4 (a) Schematic representation of the micro-cantilever bending geometry; SEM images of a micro-cantilever prepared from $\mathrm{SiC}$ matrix in $\mathrm{SiC}_{\mathrm{f}} / \mathrm{SiC}$ composites before (a) and after (b) testing ${ }^{[11,18]}$ 


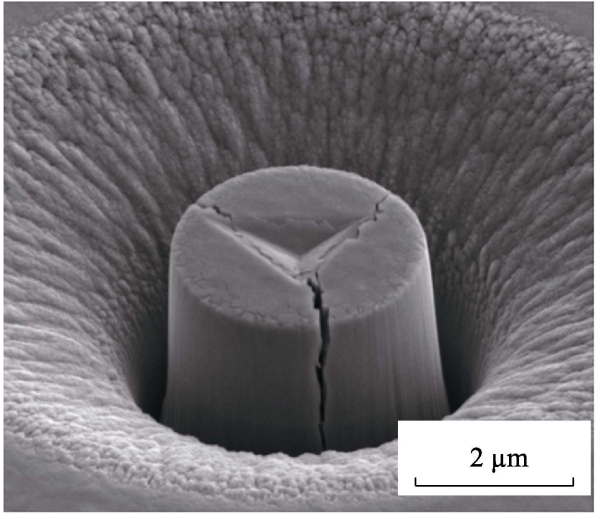

图 5 韧性测试失效微柱的 SEM 照片 ${ }^{[17]}$

Fig. 5 SEM image of an example of a pillar after splitting ${ }^{[17]}$

以消除各组分之间影响，可准确获得各组分的原位 断裂韧性数据。微柱法上述优点使之成为 CFRCMCs 各组分断裂韧性研究的有力工具。

\section{2 微柱法测量纤维与基体原位断裂韧性的 典型应用}

国防科技大学刘海蹈课题组采用微柱法率先对 PIP 工艺制备的 $\mathrm{SiC}_{\mathrm{f}} / \mathrm{SiC}$ 复合材料纤维与基体原位 断裂韧性开展了研究工作 ${ }^{[28]}$, 重点研究了裂解温度 (800、900、 $1000^{\circ} \mathrm{C}$ ) 对纤维与基体原位断裂韧性的 影响。具体过程如下: 首先采用 FIB 制备直径约为 $3 \mu \mathrm{m}$ 、长径比 1 的纤维与基体微柱(见图 6(a) (b)), 然后采用尖压头对微柱进行加载直致破坏(见图 6(c) (d)), 获得最大失效载荷 $P_{\mathrm{c}}$ (见图 6(e))。式(4)中 的 $\gamma$ 系数通过有限元模拟获得, 具体参考文献 $[28,34]$ 。 获得的不同温度制备的 $\mathrm{SiC}_{\mathrm{f}} / \mathrm{SiC}$ 复合材料中碳化硅 纤维与基体的韧性数据见图 6(f), 可以发现, 制备 温度对碳化硅纤维的断裂韧性没有明显影响, 这是 因为碳化硅纤维的制备温度一般在 $1300^{\circ} \mathrm{C}$ 以上, 相 对低的工艺温度对碳化硅纤维的组成与结构无明显 影响, 这与 KD 碳化硅纤维宏观性能分析结果一致 ${ }^{[35]}$; 测得的碳化硅纤维的原位断裂韧性约为 $2.16 \mathrm{MPa} \cdot \mathrm{m}^{1 / 2}$, 与文献[30]采用纤维切口法测量的 Tyranno SA3 的 断裂韧性相当。但碳化硅基体的断裂韧性受制备温 度影响比较显著, 由 $800^{\circ} \mathrm{C}$ 制备温度的 $2.4 \mathrm{MPa} \cdot \mathrm{m}^{1 / 2}$ 降至 $1000^{\circ} \mathrm{C}$ 的 $1.2 \mathrm{MPa} \cdot \mathrm{m}^{1 / 2}$, 说明碳化硅基体的结 晶程度显著提高, 这与 XRD 的分析结果相一致。

\section{3 界面结合强度}

界面相是 CFRCMCs 中与纤维和基体具有同等 重要地位的组分, 其性能决定着 CFRCMCs 的断裂 行为。界面相的重要功能是调控界面结合强度, 避 免过高的界面结合强度使复合材料脆性断裂, 并防
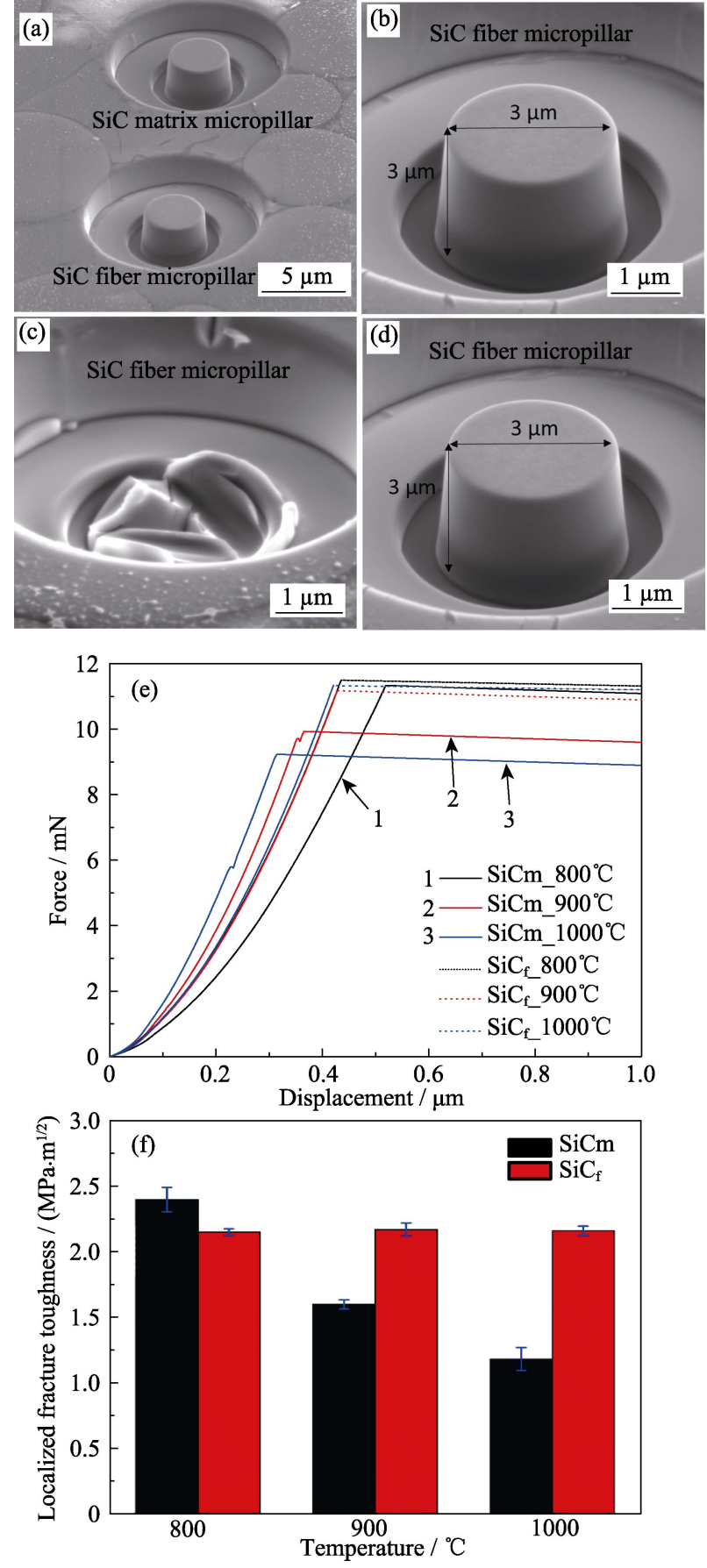

图 6 (a), (b)SiC 纤维与基体微柱; (c), (d)SiC 纤维与基体微 柱失效的 SEM 照片; (e)典型 $\mathrm{SiC}$ 纤维与基体微柱载荷-位移 曲线; (f)不同温度制备的复合材料 $\mathrm{SiC}$ 纤维与基体韧性 ${ }^{[28]}$

Fig. 6 Morphologies of the micropillars on individual SiC matrix and $\mathrm{SiC}$ fiber ( $\mathrm{a}, \mathrm{b}$ ); Micropillar morphologies of $\mathrm{SiC}$ fiber and $\mathrm{SiC}$ matrix after pillar splitting tests(c, d); Representive force-displacement curves of $\mathrm{SiC}$ matrix and $\mathrm{SiC}$ fiber by the micropillar splitting tests (e); Evolution of localized fracture toughness of the $\mathrm{SiC}$ matrix and $\mathrm{SiC}$ fiber as a function of composite fabrication temperature $(\mathrm{f})^{[28]}$

止过低的界面结合强度导致载荷无法有效传递 ${ }^{[1-3,9]}$ 。 界面结合强度是构建复合材料界面特性与宏观力学 性能的桥梁，也是指导复合材料界面相制备与工艺 优化的风向标，使界面结合强度成为 CFRCMCs 微 
观力学研究的重要内容。

\section{1 界面结合强度测量}

目前 CFRCMCs 界面结合强度的主要测量方法 包括复合材料断口拔出纤维长度统计、裂纹统计、 单纤维顶出(Fiber push-out)与单纤维顶入(Fiber pushin)四种方法。

\subsection{1 断口拔出纤维长度统计方法}

断口拔出纤维长度统计方法是最为简单的界面 结合强度评估方法, 界面结合强度 $\tau$ 可以根据式(5) 计算。其中, $S_{0}$ 和 $m$ 分别代表 CFRCMCs 中纤维原 位强度的 Weibull 参数, $r$ 为纤维半径, $h$ 为纤维拔出 长度的统计值 ${ }^{[36-38]}$ 。

$$
\tau=\frac{r \lambda(m) S_{0}}{4 h} \quad \lambda(m) \approx 0.716+\frac{1.36}{m^{0.6}}
$$

通过式(5)发现该方法存在以下问题：(1)由于 CFRCMCs 纤维难以剥离, 纤维原位强度的 Weibull 参数测试困难, 一般用原始纤维强度的 Weibull 参 数代替, 导致评估的界面结合强度准确性低; (2)断 口纤维拔出长度的统计工作量大, 主观随意性强, 数据准确性不高。因此, 该方法一般作为界面结合 强度定性分析方法。

\subsection{2 裂纹统计方法}

裂纹统计方法一般适用于束丝复合材料(Minicomposites), 包括裂纹数量统计和饱和裂纹间距统 计两种方法。

\section{(1)裂纹数量统计法}

裂纹数量统计法即对束丝复合材料进行循环加 载, 利用束丝失效前最后一个加载-卸载回线的宽 度 $(\delta \Delta)$ 和失效后测试跨距范围内的基体裂纹数量 $(N)$ 计算界面结合强度, 计算公式为 ${ }^{[39-41]}$.

$$
\begin{gathered}
\tau=\frac{b_{2} N\left(1-a_{1} V_{\mathrm{f}}\right)^{2} R_{\mathrm{f}}}{2 V_{\mathrm{f}}^{2} E_{\mathrm{m}}}\left(\frac{\sigma_{\mathrm{p}}}{\delta \Delta}\right)\left[\frac{\sigma}{\sigma_{\mathrm{p}}\left(1-\sigma / \sigma_{\mathrm{p}}\right)}\right] \\
a_{1}=E_{\mathrm{f}} / E_{\mathrm{m}} \\
b_{2}=\frac{(1+v) E_{\mathrm{m}}\left[E_{\mathrm{f}}+(1-2 v) E_{\mathrm{c}}\right]}{E_{\mathrm{f}}\left[(1+v) E_{\mathrm{f}}+(1-v) E_{\mathrm{c}}\right]}
\end{gathered}
$$

式中, $\sigma_{\mathrm{p}}$ 为初始卸载的应力; $\sigma$ 为束丝失效前最后一 个加载-卸载循环的应力; $R_{\mathrm{f}}$ 为纤维半径; $V_{\mathrm{f}}$ 为纤维 体积分数; $E_{\mathrm{f}} 、 E_{\mathrm{m}} 、 E_{\mathrm{c}}$ 分别为纤维、基体和束丝复 合材料模量; $v$ 为泊松比 (取 $v_{\mathrm{f}}=v_{\mathrm{m}}=v_{\mathrm{c}}$ )。

通过以上过程可以发现, 裂纹数量统计方法比 较复杂, 需要进行循环加载, 每个加载-卸载过程有 一定随意性; 基体裂纹数量统计具有较大的主观性, 工作量大且容易存在漏计问题。

\section{(2)裂纹间距统计法}

与裂纹数量统计法类似, 裂纹间距统计法也是
通过统计束丝复合材料失效后的裂纹计算界面结合 强度, 但过程相对简单, 直接进行加载即可, 无需 采用循环加载方式, 通过统计基体裂纹饱和空间距 离 $\left(l_{\mathrm{s}}\right)$ 计算界面结合强度 ${ }^{[39-40]}$.

$$
\tau=\frac{\sigma_{\mathrm{s}} R_{\mathrm{f}}}{2 V_{\mathrm{f}} l_{\mathrm{s}}\left(1+E_{\mathrm{f}} V_{\mathrm{f}} / E_{\mathrm{m}} V_{\mathrm{m}}\right)}
$$

式中, $\sigma_{\mathrm{s}}$ 为基体裂纹饱和时的应力, 通过载荷-位移 曲线提取; $R_{\mathrm{f}}$ 为纤维半径; $V_{\mathrm{f}}$ 为纤维体积分数; $E_{\mathrm{f}}$ 、 $E_{\mathrm{m}}$ 分别为纤维、基体模量。

尽管裂纹间距统计法加载过程较为简单，但需 要判读载荷-位移曲线(见图 7)基体裂纹饱和时的应 力, 而通过本颗题组前期的工作发现, 在具体实施 过程中, 裂纹饱和区域的判读存在较大主观因素, 并且较多样品难以呈现如图 7 所示的典型载荷-位 移曲线。另外, 与裂纹数量统计法类似, 裂纹间距统 计法也存在主观性强和不准确的问题。

\subsection{3 单纤维顶出方法}

相对以上两种方法, 单纤维顶出法是一种准确 测量复合材料界面结合强度的方法 ${ }^{[42-44]}$, 即首先制 备厚度为 $100 \mu \mathrm{m}$ 以下的复合材料薄片, 然后采用 纳米压痕(平压头)对单根纤维进行加载, 记录载荷位移曲线(见图 8), 根据式(10)计算复合材料的界面 结合强度 ${ }^{[43]}$ 。

$$
\tau=\frac{F_{\mathrm{g}}}{2 \pi R_{\mathrm{f}} l_{\mathrm{f}}}
$$

式中, $F_{\mathrm{g}}$ 为脱粘载荷, $R_{\mathrm{f}}$ 为纤维半径, $l_{\mathrm{f}}$ 为样品厚度。

单纤维顶出实验适用于各种纤维编织结构的复 合材料, 只需保证测试薄片纤维轴向与压头加载方 向平行即可。此方法计算过程简单, 各参数可准确 判读, 可以准确地测量出复合材料的界面结合强度 值, 但也存在两个主要不足: (1)CFRCMCs 的高硬

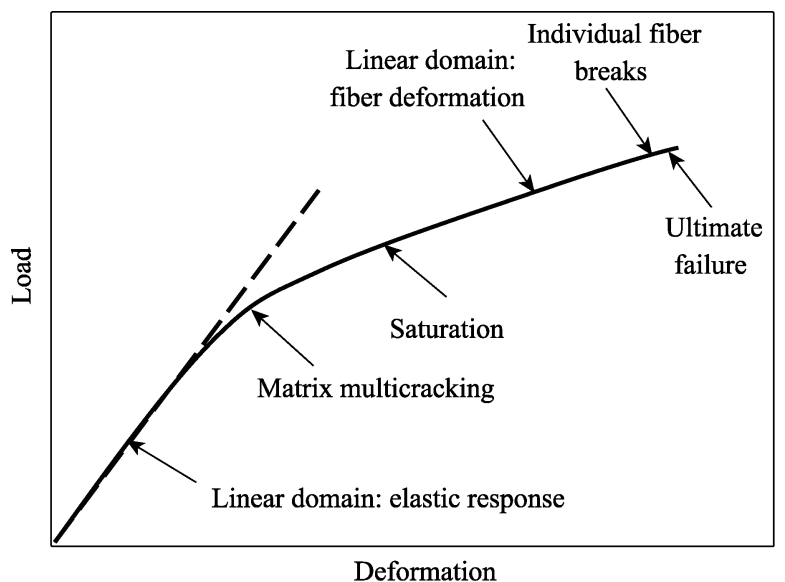

图 7 典型的束丝复合材料载荷-位移曲线 ${ }^{[39]}$

Fig. 7 Typical features of the load-deformation curve of minicomposites $^{[39]}$ 
(a)

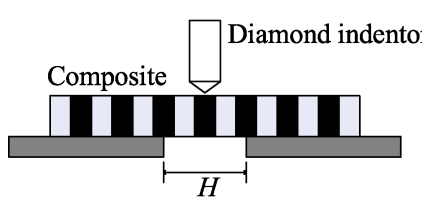

(b)
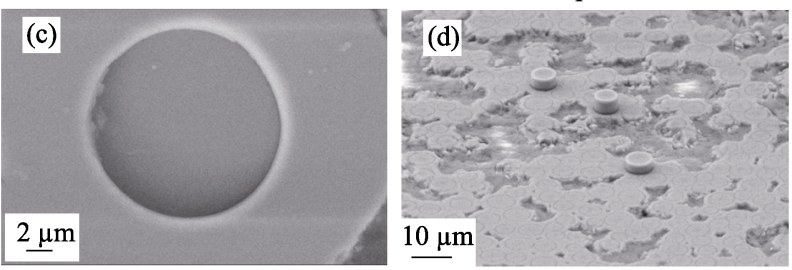

图 8 (a)单纤维顶出实验实施过程示意图; (b)典型纤维顶出 实验载荷-位移曲线; $\mathrm{SiC}_{\mathrm{f}} / \mathrm{SiC}$ 复合材料顶出纤维压头正面 $(\mathrm{c})$ 和背面(d)SEM 照片 ${ }^{[43]}$

Fig. 8 (a) Schematic drawing of fiber push-out measurement; (b) Typical load-displacement push-out test curve; SEM images of the frontside surface (c) and backside surface (d) of $\mathrm{SiC}_{\mathrm{f}} / \mathrm{SiC}$ minicomposite after fiber push-out test using a flat punch indenter ${ }^{[43]}$

度和脆性使包含数根完整纤维的薄片样品制备困难; (2)薄片在研磨制备过程中容易破坏复合材料的力学 环境, 造成界面结合强度的变化甚至界面脱粘, 影响 测试结果。以上因素使单纤维顶出方法应用越来越少。

\subsection{4 单纤维顶入方法}

单纤维顶入方法的实施过程为: 首先在复合材 料中取样，使纤维截面平行于样品表面，经抛光处 理后采用平压头对单根纤维进行加载(见图 9(a)), 记录载荷-位移曲线。典型载荷-位移曲线呈现 “ $S$ ” 型(见图 9(b)), 曲线初始段对应压头和纤维的接触 过程, 中间的线性段(斜率为 $S_{0}$ ) 对应纤维的弹性变 形，后面的曲线段对应纤维/基体界面脱粘过程 ${ }^{[45]}$ 。

根据 Shear-lag 模型即可计算复合材料的界面结合 强度 ${ }^{[10-19,28,34,45-46]}$.

$$
\tau=\frac{S_{0} P_{\mathrm{c}}}{2 \pi^{2} R_{\mathrm{f}}{ }^{3} E_{\mathrm{f}}}
$$

式中, $R_{\mathrm{f}}$ 为纤维半径; $P_{\mathrm{c}}$ 为临界载荷, 表征界面脱粘 的开始(见图 9(b)); $E_{\mathrm{f}}$ 为纤维模量。

通过以上测试过程可以发现, 单纤维顶入方法 可以准确测量界面结合强度, 相对于单纤维顶出方 法, 无需制备小厚度样品, 避免了小厚度样品制备 过程对界面力学环境的影响问题, 并且实施过程简 单。以上优点使单纤维顶入方法成为 CFRCMCs 界 面结合强度测量的有力工具。

\section{2 单纤维顶入方法测量界面结合强度的典 型应用}

国防科技大学刘海蹈课题组采用单纤维顶入方

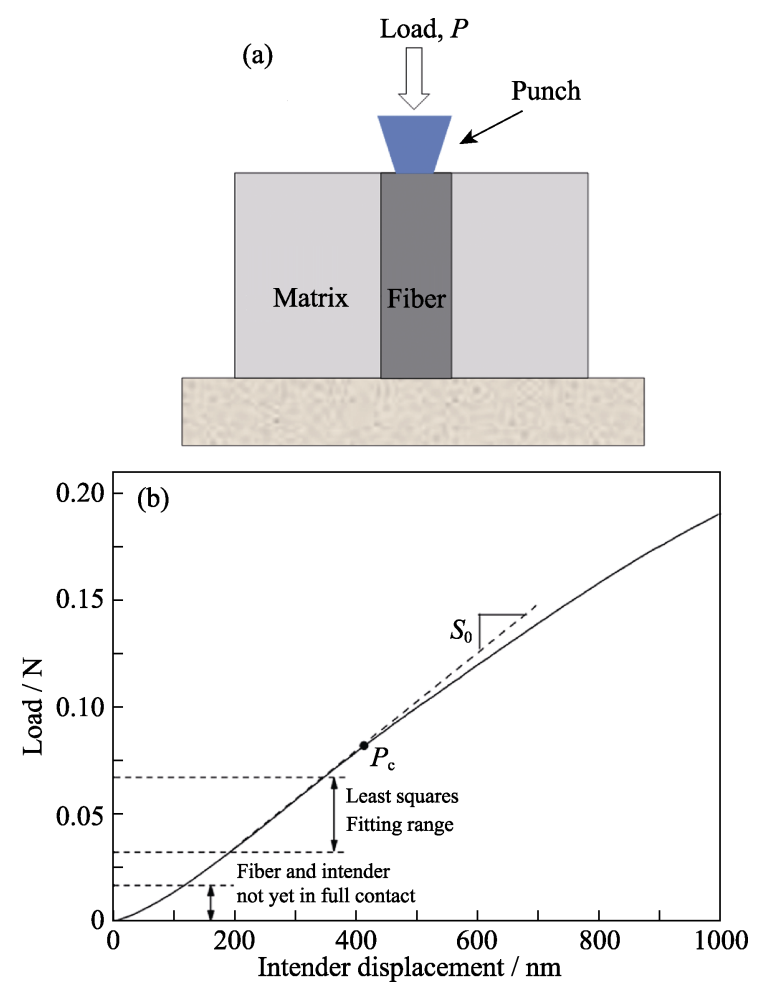

图 9 (a)纤维顶入测试示意图和(b)典型纤维顶入实验载荷位移曲线 ${ }^{[45]}$

Fig. 9 (a) Schematic drawing of fiber push-in measurement, (b) Typical load-displacement push-in test curve ${ }^{[45]}$

法开展了大量 CFRCMCs 界面结合强度的研究工作, 详细情况见表 1 。由表可见, 采用单纤维顶入方法可 以对多种类型的 CFRCMCs 界面结合强度进行表征, 界面相的引入可以有效弱化界面结合强度，使 CFRCMCs 由脆性断裂转化为㓞性断裂模式, 界面 结合强度的测试结果可以较好的解释材料的宏观力 学行为。

此外, 采用单纤维顶入方法还可以对 CFRCMCs 界面裂纹的传播模式和路径进行分析，直观揭示界 面解离机制。以本课题组研究的 Nextel440 $\mathrm{AS}_{\mathrm{f}} / \mathrm{SiC}$ 和 Nextel440 $\mathrm{AS}_{\mathrm{f}} / \mathrm{C} / \mathrm{SiC}$ 复合材料为例 ${ }^{[34]}$, 实施单纤 维顶入实验后的形貌见图 10, 由图可见，对于界面 结合强度较高的 $\mathrm{AS}_{\mathrm{f}} / \mathrm{SiC}$ 复合材料(图 10(a), 界面结 合强度见表 1)，裂纹贯穿基体，裂纹无法在界面发 生偏转; 而对于 Nextel440 $\mathrm{AS}_{\mathrm{f}} / \mathrm{C} / \mathrm{SiC}$ 复合材料(图 10(b), 界面结合强度见表 1), 由于裂解碳界面相的 引入弱化了界面结合强度, 裂纹可在界面区域发生 偏转, 并且可以观察到界面解离主要发生在纤维与 裂解碳界面相的界面，从而对界面解离机制给予直 观的解释。

\section{4 基于微观力学参数的 CFRCMCs 宏 观力学行为预判}

He-Hutchinson(简称 $\mathrm{H}-\mathrm{H}$ )模型是上世纪八九十 
表 1 国防科技大学刘海蹈课题组 CFRCMCs 界面结合强度研究工作

Table 1 Interfacial bonding strength of typical CFRCMCs investigated in our research group

\begin{tabular}{|c|c|c|c|c|c|}
\hline Composites & Interphase & $\tau / \mathrm{MPa}$ & Flexural strength/MPa & Fracture mode & Ref. \\
\hline \multirow{2}{*}{ PIP $3 \mathrm{D} \mathrm{C} / \mathrm{SiC}$} & None & 105 & 23 & Brittle & \multirow{2}{*}[19]{} \\
\hline & $\mathrm{PyC}$ & 30 & 378 & Toughened & \\
\hline \multirow{2}{*}{ PIP 3D Nextel440 $\mathrm{AS}_{\mathrm{f}} / \mathrm{SiC}$} & None & 293 & 45 & Brittle & \multirow{2}{*}[34]{} \\
\hline & $\mathrm{PyC}$ & 42 & 163 & Toughened & \\
\hline \multirow{2}{*}{ Sol-Gel 3D SiC $\mathrm{f} /$ Mullite } & None & 537 & 230 & Brittle & \multirow{2}{*}[46]{} \\
\hline & $\mathrm{PyC}$ & 155 & 35 & Toughened & \\
\hline \multirow{2}{*}{ PIP 3D $\mathrm{SiC}_{\mathrm{f}} / \mathrm{SiC}$} & None & 450 & 90 & Brittle & \multirow{2}{*}[28]{} \\
\hline & $\mathrm{BN}$ & 50 & 200 & Toughened & \\
\hline Sol-Gel 3D ALF $\mathrm{AS}_{\mathrm{f}} / \mathrm{SiO}_{2}\left(600^{\circ} \mathrm{C}\right)$ & None & 50 & 105 & Toughened & \multirow{2}{*}[10]{} \\
\hline Sol-Gel 3D ALF AS $\mathrm{A}_{\mathrm{f}} / \mathrm{SiO}_{2}\left(1200^{\circ} \mathrm{C}\right)$ & None & 260 & 45 & Brittle & \\
\hline
\end{tabular}

*PIP: Precursor infiltration and pyrolysis; $\mathrm{AS}_{\mathrm{f}}$ : Aluminosilicate fiber
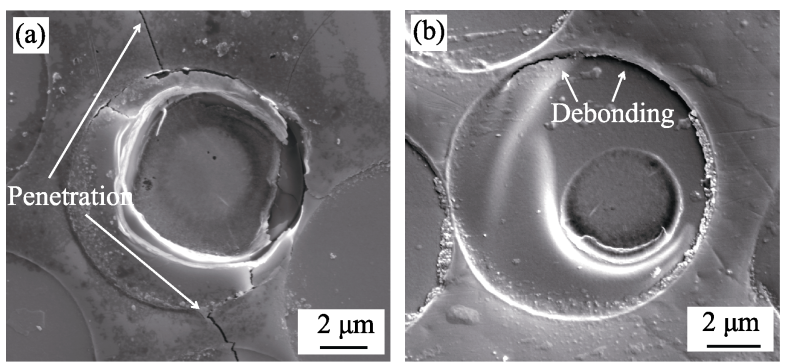

图 10 单纤维顶入实验后 $\mathrm{AS}_{\mathrm{f}} / \mathrm{SiC}(\mathrm{a})$ 和 $\mathrm{AS}_{\mathrm{f}} / \mathrm{C} / \mathrm{SiC}$ (b)复合 材料压入纤维的 SEM 照片 ${ }^{[34]}$

Fig. 10 SEM images of the pushed fiber in $\mathrm{AS}_{\mathrm{f}} / \mathrm{SiC}$ (a) and $\mathrm{AS}_{\mathrm{f}} / \mathrm{C} / \mathrm{SiC}(\mathrm{b})$ composites $^{[34]}$

年代发展起来的针对 CFRCMCs 宏观力学行为预判 的重要方法 ${ }^{[12,47-49]}$, 根据 CFRCMCs 的微观力学参 数可以预判裂纹传播模式(贯穿或偏转), 且易于实 施。但由于以前无法准确测量微观力学参数, 仅能 采用各组分的估算值或宏观力学参数代替原位微观 力学参数, 往往造成误判。CFRCMCs 微观力学研究 工作的进展, 使 H-H 模型预判 CFRCMCs 的宏观力 学行为的准确度显著提高。

根据 H-H 模型, CFRCMCs 裂纹在界面发生偏 转需满足式(12):

$$
\frac{\Gamma_{\mathrm{i}}}{\Gamma_{\mathrm{f}}}<\frac{G_{\mathrm{d}}}{G_{\mathrm{p}}}
$$

式中, $\Gamma_{\mathrm{i}}$ 和 $\Gamma_{\mathrm{f}}$ 分别为界面和纤维韧性; $G_{\mathrm{d}}$ 和 $G_{\mathrm{p}}$ 分别 代表裂纹偏转和贯穿的能量释放速率, 两者比值可 表征为:

$$
\frac{G_{\mathrm{d}}}{G_{\mathrm{p}}}=\frac{1}{4(1-\alpha)^{0.9}}
$$

式中, $\alpha$ 为弹性失配系数，可近似表征为:

$$
\alpha=\frac{E_{\mathrm{f}}-E_{\mathrm{m}}}{E_{\mathrm{f}}+E_{\mathrm{m}}}
$$

此外, $\Gamma_{\mathrm{i}}$ 与基体韧性 $\Gamma_{\mathrm{m}}$ 一般呈线性关系, 出于 保守设计, 可用 $\Gamma_{\mathrm{m}}$ 代替 $\Gamma_{\mathrm{i}}$ 。

复合材料各组分的韧性 $(\Gamma)$ 可由断裂韧性 $K_{\mathrm{IC}}$ 根 据公式(15)计算得到, 其中 $K_{\mathrm{IC}}$ 可采用第 2 部分介绍 方法测量获得。

$$
\Gamma=K_{\mathrm{IC}}^{2} / E
$$

根据 $\mathrm{H}-\mathrm{H}$ 模型，本课题组对不同温度制备的 $\mathrm{PIP} \mathrm{SiC}_{\mathrm{f}} / \mathrm{SiC}$ 以及 $\mathrm{SiC}_{\mathrm{f}} / \mathrm{BN} / \mathrm{SiC}$ 复合材料的宏观力学 行为进行了预判, 各组分微观力学参数以及 $\mathrm{H}-\mathrm{H}$ 模 型预判结果分别见表 2 和图 $11^{[28]}$ 。由图可见, 采用 $\mathrm{H}-\mathrm{H}$ 模型预判 $\mathrm{SiC}_{\mathrm{f}} / \mathrm{SiC}$ 复合材料裂纹以贯穿模式传 播, 复合材料呈现脆性断裂行为, 这主要由 $\mathrm{SiC}_{\mathrm{f}} / \mathrm{SiC}$ 复 合材料弹性不匹配系数较小、且界面韧性较高所致; 而对于 $\mathrm{SiC}_{\mathrm{f}} / \mathrm{BN} / \mathrm{SiC}$ 复合材料, 由于低模量和低韧

表 2 国防科技大学刘海蹈课题组研究的 $\mathrm{SiC}_{\mathrm{f}} / \mathrm{SiC}$ 和 $\mathrm{SiC}_{\mathrm{f}} / \mathrm{BN} / \mathrm{SiC}$ 复合材料微观力学参数 ${ }^{[28]}$

Table 2 Micro-mechanical parameters of $\mathrm{SiC}_{\mathrm{f}} / \mathrm{SiC}$ and $\mathrm{SiC}_{\mathrm{f}} / \mathrm{BN} / \mathrm{SiC}$ composites investigated in Liu's group ${ }^{[28]}$

\begin{tabular}{ccccccc}
\hline Composites & $E_{\mathrm{m}} / \mathrm{GPa}$ & $E_{\mathrm{f}} / \mathrm{GPa}$ & $\Gamma_{\mathrm{m}} /\left(\mathrm{J} \cdot \mathrm{m}^{-2}\right)$ & $\Gamma_{\mathrm{f}} /\left(\mathrm{J} \cdot \mathrm{m}^{-2}\right)$ & $E_{\mathrm{BN} \text { interphase }} / \mathrm{GPa}$ & $\Gamma_{\mathrm{BN} \text { interphase }} /\left(\mathrm{J} \cdot \mathrm{m}^{-2}\right)$ \\
\hline $\mathrm{SiC}_{\mathrm{f}} / \mathrm{SiC}\left(800^{\circ} \mathrm{C}\right)$ & 118 & 160 & 49 & 29 & - & - \\
$\mathrm{SiC}_{\mathrm{f}} / \mathrm{SiC}\left(900^{\circ} \mathrm{C}\right)$ & 170 & 160 & 15 & 29 & - & - \\
$\mathrm{SiC}_{\mathrm{f}} / \mathrm{SiC}\left(1000^{\circ} \mathrm{C}\right)$ & 256 & 160 & 5 & 29 & - & - \\
$\mathrm{SiC}_{\mathrm{f}} / \mathrm{BN} / \mathrm{SiC}$ & - & 160 & - & 29 & 70 & 4 \\
\hline
\end{tabular}




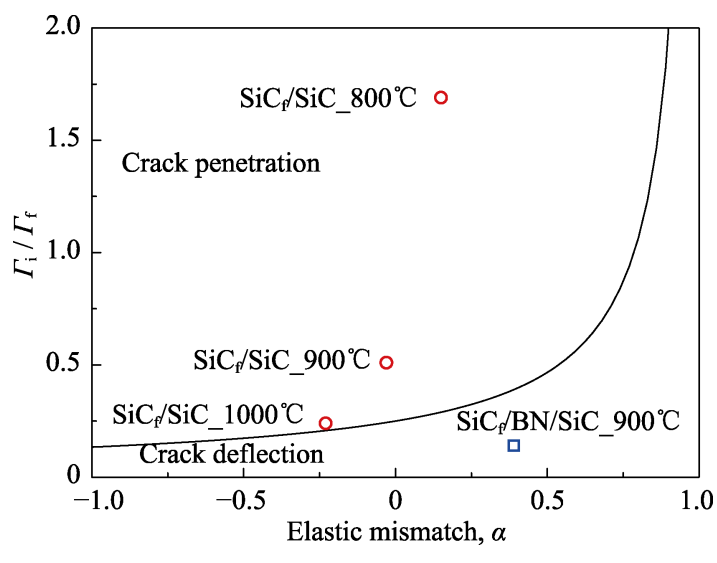

图 11 基于 $\mathrm{H}-\mathrm{H}$ 模型的 $\mathrm{SiC}_{\mathrm{f}} / \mathrm{SiC}$ 和 $\mathrm{SiC}_{\mathrm{f}} / \mathrm{BN} / \mathrm{SiC}$ 复合材料 宏观力学行为预判 ${ }^{[28]}$

Fig. 11 Predictions on macro-mechanical behavior of $\mathrm{SiC}_{\mathrm{f}} / \mathrm{SiC}$ and $\mathrm{SiC}_{\mathrm{f}} / \mathrm{BN} / \mathrm{SiC}$ composites based on $\mathrm{H}-\mathrm{H}$ model ${ }^{[28]}$

性 BN 界面相的引入(见表 2), 增大了弹性不匹配系 数, 并降低了界面与纤维的韧性比, 使复合材料的 裂纹表现为偏转传播模式, 复合材料呈现韧性断裂 行为。以上理论预判结果与宏观力学性能测试结果 相吻合 ${ }^{[28]}$ 。需要补充说明的是, 以上讨论过程并未 考虑 $\mathrm{SiC}_{\mathrm{f}} / \mathrm{SiC}$ 复合材料可能存在的界面反应情况。

因此, 采用准确的微观力学参数可以利用成熟 的 H-H 模型对 CFRCMCs 的宏观力学行为给予很好 的预判，这对于 CFRCMCs 的组分设计与工艺优化 具有较强的理论指导意义。

\section{5 结语}

借助于日趋完善的基于纳米压痕的微观力学测 试方法和基于 FIB 的微尺度测量样品的制备方法, 可以有效解决长期困扰 CFRCMCs 研究人员的微观 力学参数测量问题，这将极大促进 CFRCMCs 仿真、 机理分析、工艺优化等工作的研究进展, 有望改变 目前 CFRCMCs 主要依靠宏观力学实验开展研究工 作的现状。另外, 微观力学参数可以有效构建 CFRCMCs 组分、工艺、宏观力学性能间的内在联 系, 破解目前 CFRCMCs 设计性差、机理阐述不清 晰的难题。但 CFRCMCs 微观力学研究工作尚在发 展之中，诸如高温原位微观力学参数测试技术、界 面韧性测试方法等研究工作还有相当长的一段路需 要走。相信通过各相关领域研究人员的努力, 最终 将促进 CFRCMCs 按需定制、多尺度量化设计局面 的形成。

\section{参考文献:}

[1] 陈朝辉, 李 伟, 王 松, 等. 先驱体转化陶瓷基复合材料, 北京: 科学出版社, 2012 .
[2] 张立同. 纤维增㓞碳化硅陶瓷复合材料一模拟、表征与设计. 北 京: 化学工业出版社, 2009 .

[3] KRENKEL WALTER. Ceramic Matrix Composites. Weinheim: WILEY-VCH Verlag GmbH \& Co. KGaA, 2008.

[4] WANG YI, LIU HAI-TAO, CHENG HAI-FENG. Research progress on oxide/oxide ceramic matrix composites. Journal of Inorganic Materials, 2014, 29(7): 673-680.

[5] MA QING-SONG, LIU HAI-TAO, PAN YU, et al. Research progress on the application of $\mathrm{C} / \mathrm{SiC}$ composites in scramjet. Journal of Inorganic Materials, 2013, 28(3): 247-255.

[6] MEYER P, WAAS A M. FEM predictions of damage in continuous fiber ceramic matrix composites under transverse tension using the crack band method. Acta Materialia, 2016, 102: 292-303.

[7] Li L B, SONG Y D, SUN Y C. Modeling the tensile behavior of unidirectional $\mathrm{C} / \mathrm{SiC}$ ceramic-matrix composites. Mechanics of Composite Materials, 2014, 49(6): 659-672.

[8] WANG L, WANG Z, DONG S M, et al. Finite element simulation of stress distribution and development of $\mathrm{C}_{\mathrm{f}} / \mathrm{SiC}$ ceramice-matrix composite coated with single layer $\mathrm{SiC}$ coating during thermal shock. Composites: Part B, 2013, 51: 204-214.

[9] EVANS A G, ZOK F W. The physics and mechanics of fibre-reinforced brittle matrix composites. Journal of Materials Science, 1994, 29: 3857-3896.

[10] YANG L W, WANG J Y, LIU H T, et al. Sol-Gel temperature dependent ductile-to-brittle transition of aluminosilicate fiber reinforced silica matrix composite. Composites: Part B, 2017, 119: $79-89$.

[11] FRAZER D, ABAD M D, KRUMWIEDE D, et al. Localized mechanical property assessment of $\mathrm{SiC} / \mathrm{SiC}$ composite materials. Composites: Part A, 2015, 70: 93-101.

[12] BLAESE D, GARCIA D E, GUGLIELMI P, et al. $\mathrm{ZrO}_{2}$ fibermatrix interfaces in alumina fiber-reinforced model composites. Journal of European Ceramic Society, 2015, 35: 1593-1598.

[13] UDAYAKUMAR A, SRI GANESH A, RAJA S, et al. Effect of intermediate heat treatment on mechanical properties of $\mathrm{SiC}_{\mathrm{f}} / \mathrm{SiC}$ composites with $\mathrm{BN}$ interphase prepared by ICVI. Journal of the European Ceramic Society, 2011, 31: 1145-1153.

[14] YAN W, PUN C L, WU Z, et al. Some issues on nanoindentation method to measure the elastic modulus of particles in composites. Composites: Part B, 2011, 42: 2093-2097.

[15] ZHANG L, REN C, ZHOU C, et al. Single fiber push-out characterization of interfacial mechanical properties in unidirectional CVI-C/SiC composites by the nano-indentation technique. Applied Surface Science, 2015, 357: 1427-1433.

[16] HINOKI T, ZHANG W, KOHYAMA A, et al. Effect of fiber coating on interfacial shear strength of $\mathrm{SiC} / \mathrm{SiC}$ by nano-indentation technique. Journal of Nuclear Materials, 1998, 258-263: 15671571.

[17] SEBASTIANI M, JOHANNS K E, HERBERT E G, et al. A novel pillar indentation splitting test for measuring fracture toughness of thin ceramic coatings. Philosophical Magazine, 2015, 95: 19281944.

[18] SEBASTIANi M, JOHANNS K E, HERBERT E G, et al. Measurement of fracture toughness by nanoindentation methods: recent advances and future challenges. Current Opinion in Solid State and Materials Science, 2015, 19: 324-333.

[19] LIU H T, YANG L W, SUN X, et al. Enhancing the fracture resistance of carbon fiber reinforced $\mathrm{SiC}$ matrix composites by interface modification through a simple fiber heat-treatment process. Carbon, 2016, 109: 435-443.

[20] LEATHERBARROW A, WU H Z. Mechanical behaviour of the 
constituents inside carbon-fibre/carbon-silicon carbide composites characterised by nano-indentation. Journal of the European Ceramic Society, 2012, 32: 579-588.

[21] MARX D T, RIESTER L. Mechanical properties of carbon-carbon composite components determined using nanoindentation. Carbon, 1999, 37: 1679-1684.

[22] DISS P, LAMON J, CARPENTIER L, et al. Sharp indentation behavior of carbon/carbon composites and varieties of carbon. Carbon, 2002, 40: 2567-2579.

[23] MULLER W M, MOOSBURGER-WILL J, SAUSE M G R, et al. Quantification of crack area in ceramic matrix composites at singlefiber push-out testing and influence of pyrocarbon fiber coating thickness on interfacial fracture toughness. Journal of the European Ceramic Society, 2015, 35: 2981-2989.

[24] OLIVER W C, PHARR G M. An improved technique for determining hardness and elastic modulus using load and displacement sensing indentation experiments. Journal of Materials Research, 1992, 7(6): 1564-1583.

[25] VANLANDINGHAM M R. Review of instrumented indentation. Journal of Research of the National Institute of Standards and Technology, 2003, 108(4): 249-265.

[26] OLIVER W C, PHARR G M. Measurement of hardness and elastic modulus by instrumented indentation: advances in understanding and refinements to methodology. Journal of Materials Research, 2004, 19(1): 3-20.

[27] FISCHER-CRIPPS A C. Critical review analysis and interpretation of nanoindentation test data. Surface \& Coatings Technology, 2006, 200: 4153-4165.

[28] YANG L W, LIU H T, CHENG H F. Processing-temperature dependent micro- and macro-mechanical properties of SiC fiber reinforced SiC matrix composites. Composites: Part B, 2017, 129: $152-161$.

[29] HONJO K. Fracture toughness of PAN-based carbon fibers estimated from strength-mirror size relation. Carbon, 2003, 41: 979984.

[30] MORISHITA K, OCHIAI S, OKUDA H, et al. Fracture toughness of a crystalline silicon carbide fiber (tyranno-SA $3^{\circledR}$ ). Journal of the American Ceramic Society, 2006, 89(8): 2571-2576.

[31] OCHIAI S, KUBOSHIMA S, MORISHITA K, et al. Fracture toughness of $\mathrm{Al}_{2} \mathrm{O}_{3}$ fibers with an artificial notch introduced by a focused-ion-beam. Journal of the European Ceramic Society, 2010, 30: $1659-1667$.

[32] CASEllaS D, CARO J, MOLAS S, et al. Fracture toughness of carbides in tool steels evaluated by nanoindentation. Acta Materialia, 2007, 55: 4277-4286.

[33] MUELLER M G, PEJCHAL V, ŽAGAR G, et al. Fracture toughness testing of nanocrystalline alumina and fused quartz using chevron-notched microbeams. Acta Materialia, 2015, 86: 385-395.

[34] LIU H T, YANG L W, HAN S, et al. Interface controlled microand macro-mechanical properties of aluminosilicate fiber reinforced SiC matrix composites. Journal of the European Ceramic Society, 2017, 37: 883-890.

[35] CAO S Y, WANG J, WANG H. High-temperature behavior and degradation mechanism of $\mathrm{SiC}$ fibers annealed in $\mathrm{Ar}$ and $\mathrm{N}_{2}$ atmospheres. Journal of Materials Science, 2016, 51: 4650-4659.

[36] DAVIES I J, ISHIKAWA T, SHIBUYA M, et al. Fibre and interfa- cial properties measured in situ for a 3D woven $\mathrm{SiC} / \mathrm{SiC}$-based composite with glass sealant. Composites: Part A, 1999, 30: 587591.

[37] DAVIES I J , OGASAWARA T, ISHIKAWA T. Distribution of fibre pullout length and interface shear strength within a single fibre bundle for an orthogonal 3-D woven Si-Ti-C-O fibre/Si-Ti-C-O matrix composite tested at $1100^{\circ} \mathrm{C}$ in air. Journal of the European Ceramic Society, 2005, 25: 599-604.

[38] BRANDSTETTER J, PETERLIK H, KROMP K, et al. A new fibrebundle pull-out test to determine interface properties of a $2 \mathrm{D}$ - woven carbon/carbon composite. Composites Science Technology, 2003, 63: 653-660.

[39] BERTRAND S, FORIO P, PAILLER R, et al. Hi-Nicalon/SiC minicomposites with (pyrocarbon/ $\mathrm{SiC}$ ) ${ }_{\mathrm{n}}$ nanoscale multilayered interphases. Journal of the American Ceramic Society, 1999, 82(9): 2465-2473.

[40] SAUDER C, BRUSSON A, LAMON J. Influence of interface characteristics on the mechanical properties of Hi-Nicalon type-S or Tyranno-SA3 fiber-reinforced $\mathrm{SiC} / \mathrm{SiC}$ minicomposites. International Journal of Applied Ceramic Technology, 2010, 7(3): 291303.

[41] MORSCHER G N, MARTINEZ-FERNANDEZ J. Fiber effects on minicomposite mechanical properties for several silicon carbide fiber-chemically vapor-infiltrated silicon carbide matrix systems. Journal of the American Ceramic Society, 1999, 82(1): 145-155.

[42] REBILLAT F, LAMON J, GUETTE A. The concept of a strong interface applied to $\mathrm{SiC} / \mathrm{SiC}$ composites with a $\mathrm{BN}$ interphase. Acta Materialia, 2000, 48: 4609-4618.

[43] BUET E, SAUDER C, SORNIN D, et al. Influence of surface fibre properties and textural organization of a pyrocarbon interphase on the interfacial shear stress of $\mathrm{SiC} / \mathrm{SiC}$ minicomposites reinforced with Hi-Nicalon S and Tyranno SA3 fibres. Journal of the European Ceramic Society, 2014, 34: 179-188.

[44] MUELLER W M, MOOSBURGER-WILL J, SAUSE M G R, et al. Microscopic analysis of single-fiber push-out tests on ceramic matrix composites performed with Berkovich and flat-end indenter and evaluation of interfacial fracture toughness. Journal of the European Ceramic Society, 2013, 33: 441-451.

[45] RODRÍGUEZ M, MOLINA-ALDAREGUÍA J M, GONZÁLEZ C, et al. A methodology to measure the interface shear strength by means of the fiber push-in test. Composites Science and Technology, 2012, 72: 1924-1932.

[46] HAN S, YANG L W, LIU H T, et al. Micro-mechanical properties and interfacial engineering of $\mathrm{SiC}$ fiber reinforced Sol-Gel fabricated mullite matrix composites. Materials and Design, 2017, 131: $265-272$.

[47] HE M Y, HUTCHINSON J W. Crack deflection at the interface between dissimilar materials. International Journal of Solids and Structures, 1989, 25(9): 1053-1067.

[48] HE M Y, EVANS A G, HUTCHINSON J W. Crack deflection at an interface between dissimilar elastic materials: role of residual stresses. International Journal of Solids and Structures, 1994, 31(24): 3443-3455.

[49] FUJITA H, JEFFERSON G, MCMEEKING R M, et al. Mullite/alumina mixtures for use as porous matrices in oxide fiber composites. Journal of the American Ceramic Society, 2004, 87(2): 261-267. 